\title{
Linx
}

Revue des linguistes de l'université Paris X Nanterre

12 | 2002

«Comme la lettre dit la vie»

\section{Un cas de double incise de discours rapporté}

\section{Annie Delaveau}

\section{OpenEdition}

\section{Journals}

Édition électronique

URL : http://journals.openedition.org/linx/1279

DOI : $10.4000 /$ linx. 1279

ISSN : 2118-9692

\section{Éditeur}

Presses universitaires de Paris Nanterre

\section{Édition imprimée}

Date de publication : 1 octobre 2002

Pagination : 64-70

ISSN : 0246-8743

\section{Référence électronique}

Annie Delaveau, « Un cas de double incise de discours rapporté », Linx [En ligne], 12 | 2002, mis en ligne le 10 octobre 2012, consulté le 19 avril 2019. URL : http://journals.openedition.org/linx/1279 ; DOI : 10.4000/linx.1279

Ce document a été généré automatiquement le 19 avril 2019.

Département de Sciences du langage, Université Paris Ouest 


\title{
Un cas de double incise de discours rapporté
}

\author{
Annie Delaveau
}

1 Il s'agit d'un corpus obtenu par hasard. Le hasard de lectures romanesques. D'abord, il y a quelques années, en lisant La Rabouilleuse, je rencontre le premier exemple, que j'archive. Et puis la lecture du roman de Rezvani, L'Origine du monde, paru en 2000, aux éditions Actes Sud, révèle 24 exemples de la même forme, que celui de Balzac :

«- Il débouche en ce moment douze cents personnes du faubourg de Rome, vient de me dire un de mes hommes, fit observer le lieutenant de gendarmerie, et ils poussent des cris de mort. "

2 La structure du roman de Rezvani, dont l'action se déroule entre 2015 et 2020, est de la forme suivante : un narrateur, sans nom, recueille les confidences du nain Bergamme, qui est en train de mourir sur un lit d'hôpital. Celui-ci lui raconte qu'il est le voleur de tableaux de musée que toutes les polices recherchent, et comment il a réussi à gagner la confiance du conservateur du Grand Musée, Gerbraun, et du commissaire chargé de sa sécurité, Quevedo. D'autres personnages sont mis en scène dans le récit de Bergamme, dont le but, en s'installant dans le musée où il finit par vivre, au même titre que le commissaire, est de voler le tableau de Courbet, l'Origine du Monde.

3 Le texte ne comporte pratiquement pas de récit au sens où Genette utilise le terme, en reprenant les propriétés attribuées à l'énonciation historique par Benveniste, à savoir des énoncés sans je, et dont le temps-repère est le passé simple. Les événements sont racontés par la mise en scène des discours que tient et que rapporte Bergamme au narrateur qui les prend en note: Bergamme parle en son nom, et d'autres personnages prennent la parole également.

4 La forme de la double incise de discours rapporté, inattendue, est la suivante dans tous les cas relevés :

discours rapporté - incise 1- incise 2 - discours rapporté

5 Ce qu'on perçoit à première vue, c'est un enchaînement linéaire de deux incises à sujet inversé, l'une contiguë à l'autre. 
Dans toutes les occurrences, on observe les propriétés attendues de ce type d'incise : le sujet, qu'il soit pronominal ou nominal, y est inversé, ce qui permet de caractériser la forme de l'incise de discours rapporté comme une construction, au sens de la grammaire de Constructions, puisqu'on y voit fonctionner de la même façon les deux types de sujet, nominal et pronominal. Or on sait qu'il n'y a pas d'autre contexte que celui de l'incise de discours rapporté dans lequel les deux formes de sujet sont susceptibles de se comporter de la même façon, eu égard aux possibilités d'inversion.

On peut formuler deux hypothèses sur l'organisation des deux incises et leur portée :

$8 \quad 1^{\text {ère }}$ hypothèse : discours rapporté [incise 1, incise 2]

9 Selon cette hypothèse, le discours rapporté est dans la portée des deux incises. Le problème est la relation entre les deux incises : pur enchaînement, forme d'addition, chacune ayant dans sa portée le discours rapporté, il n'y a pas forcément à établir une relation entre les deux incises, si elles sont dans la même relation au discours rapporté.

11 Selon cette hypothèse, le discours rapporté est dans la portée de l'incise 1, et la forme ainsi constituée - pour laquelle on ne dispose pas pour l'instant de nom - est à son tour dans la portée de l'incise 2.

Dans ces deux hypothèses, on appelle « discours rapporté » tout ce qui a les propriétés du discours direct, à l'exclusion des incises.

On essaiera de rendre compte du phénomène en s'inspirant des hypothèses formulées par Ann Banfield (1982: 15), qui s'exprime de la façon suivante : « La littérature ne se nourrit pas d'infractions aux règles, il faut bien plutôt la concevoir comme une série d'expériences testant les limites de la langue ».

\section{Observations}

14 On commence par quelques observations qui permettent d'envisager les arguments en faveur de l'une ou l'autre hypothèse. Celles-ci portent sur la place de la double incise, sur les temps et les personnes utilisées dans les deux incises successives.

\section{La place}

15 La double incise peut être soit en fin de discours, soit insérée à l'intérieur du discours rapporté, ce qui constitue un argument en faveur de la $1^{\text {ère }}$ hypothèse, puisque la double incise occupe les deux places ouvertes à l'incise ordinaire.

Cependant l'observation des exemples (16) et (24) autorise à ne pas faire fond de cette première constatation :

16) Et c'est comme ça, m'avait dit Roberte, avait continué avec presque joyeuse humeur Bergamme, oui c'est comme ça, m'avait-elle dit, que peu à peu les parois peintes commencèrent à se couvrir d'une légère moisissure... (p. 302)

24) - C'est ce que vous auriez dû accomplir, lui avais-je répondu en grinçant d'un rire que j'entendais avec horreur sans pouvoir cependant le réprimer, continuait Bergamme, oui, avais-je dit à Gerbraun, c'est à ce moment - là qu'il était encore humainement possible de m'anéantir... (p. 397)

En (16), le début du discours rapporté suivi de l'incise 1 est répété après la double incise : «c'est comme ça, m'avait-elle dit ». En (24), l'incise 1 est également répétée après une 
reprise anaphorique du début du discours rapporté, avec une variante du verbe de l'incise ; le verbe dire reprend comme un hyperonyme le verbe répondre : « oui, avais-je dit à Gerbraun ». Ce que suggère, en effet, cette reprise ou cette répétition de l'incise 1, sans l'incise 2, c'est que les deux incises ne forment pas un bloc comme le figure la représentation proposée pour la $1^{\text {ère }}$ hypothèse, et que le discours rapporté est dans la portée étroite de l'incise 1 , mais pas de l'incise 2.

\section{Les temps}

Il y a une proportion massive d'imparfaits et de plus -que -parfaits dans l'incise 2, ainsi que dans l'incise 1, mais il n'y a pas de tendance statistique forte dans les couples de temps réalisés dans les deux incises, ni d'exclusion qui aurait quelque pertinence : ainsi on observe le passé simple aussi bien dans l'incise 1 que dans l'incise 2. Il faut donc examiner des cas que l'on peut considérer comme cruciaux.

Ceux-ci sont constitués par l'occurrence du présent (ou du passé composé), forme typique du Discours, dans l'une ou l'autre incise.

Dans l'incise 2, le présent apparaît deux fois, en (3) et en (6), et le passé composé une fois en (10) :

3) Ce qu'il n'a pas réussi, hélas! l'avais-je interrompu en jubilant, poursuit Bergamme.

(p. 117)

6) «Voyez-vous Gerbraun, avais-je continué, dit Bergamme, si Guernica n'avait pas ces

dimensions, il y a longtemps que je l'aurais volé. » (p. 119)

10) «Mais non, jeune homme, lui avait dit Gerbraun, m'a encore rapporté mon

collaborateur en hygrométrie, vous ne nous dérangez pas du tout !» (p. 170)

En (3) le présent s'interprète par embrayage sur la situation initiale du roman, le récit par le nain Bergamme au narrateur sans nom de son aventure.

En (6), on peut hésiter sur le temps de « dit Bergamme » étant donné l'homonymie de la forme verbale. Mais il apparaît qu'on doit interpréter comme dans l'exemple (3), où la forme du verbe de l'incise 2 s'interprète par embrayage sur la situation initiale du roman.

En (10), le passé composé est interprété par embrayage, également, mais cette fois sur une situation interne au roman, celle où l'hygrométreur, dit Alf le Crapaud, raconte son histoire.

Dans l'incise 1, le présent (ou le passé composé) apparaît 3 fois, en (1), (7), et (9) :

1) «-Il débouche en ce moment douze cents personnes du faubourg de Rome, vient

de me dire un de mes hommes, fit observer le lieutenant de gendarmerie, et ils poussent des cris de mort. »

7) «Bergamme est un petit homme tout à fait singulier, m'a dit Gerbraun, avait poursuivi Roberte, il veut tellement être le voleur des tableaux... » (p. 148)

9) Mais au contraire - et cela je ne l'ai compris que plus tard, a précisé mon jeune

collaborateur Félix, continuait Alfle Crapaud - d'avoir été surpris avec Elise ... (p. 169)

En (1), l'exemple de Balzac, le présent est interprété par embrayage sur l'événement décrit dans l'incise 2. De même en (7) et en (9), le passé composé est interprété par rapport à l'événement décrit par l'incise 2. Dans les trois cas, le présent ou le passé composé réfère à un moment, qui est distinct de l'événement décrit par l'incise 2. L'incise 2 est à un temps de l'Histoire, le passé simple ou l'imparfait et le plus-que-parfait, l'incise 1 est à un temps du Discours, le présent ou le passé composé. C'est là que réside l'effet de nouveauté de l'usage des temps dans la double incise, dans le fait que ce présent ou ce 
passé composé est interprétable comme un temps de Discours par rapport à un temps de l'Histoire désigné soit par le passé simple, dans l'exemple de Balzac, soit par le plus-queparfait ou l'imparfait dans les deux exemples de Rezvani. Autrement dit, la mise en relation des temps de l'incise 1 avec ceux de l'incise 2 n'est pas une relation interne au système de l'Histoire au sens de Benveniste, mais une relation asymétrique qui place l'incise 2 dans les temps de l'Histoire et l'incise 1 dans les temps du Discours.

La conséquence de cette distribution des temps est la suivante : les deux incises ne sont pas, comme le laisserait supposer la représentation attachée à la 1ère hypothèse, sur le même plan, mais plutôt, comme le laisse supposer la représentation de la $2^{\text {ème }}$ hypothèse, sur deux plans distincts. La seconde est dans le plan de l'Histoire - ou du récit au sens de Genette, l'incise 1 est dans le plan du Discours. La relation entre les deux n'est pas une relation d'équivalence, mais c'est la relation qui s'instaure couramment entre l'introducteur de discours rapporté et le discours rapporté lui-même. Ce qui est paradoxal.

\section{Les personnes}

Le paradoxe formulé à propos des relations entre les formes temporelles des deux incises dans les cas cruciaux est à la fois confirmé et résolu par l'observation des personnes dans les deux incises. On entend par "personnes » soit le sujet du verbe de communication de l'incise, soit un complément indirect de ce verbe - indiqués dans le tableau comme sujet ou coi.

A propos des personnes, on observe une nette asymétrie entre l'incise 1 et l'incise 2 . Toutes les incises 2 ont un sujet à la $3^{\text {ème }}$ personne. Dans trois cas, il y a un complément du verbe de dire à la $1^{\text {ère }}$ personne, en (10), (11) et (17) :

10) «Mais non, jeune homme, lui avait dit Gerbraun, m'a encore rapporté mon collaborateur en hygrométrie, vous ne nous dérangez pas du tout !» (p. 170)

11) «Cesse de te plaindre, Gerbraun, avait dit Elise toujours allongée sous l'édredon, m'a encore rapporté mon jeune collaborateur. (p. 171)

17) On aurait pu penser, ajoutait encore Quevedo, juste quelques instants avant que ne survienne le second « accident » du Grand Musée, m'avait dit Bergamme toujours couché sur le dos, oui, on aurait pu penser qu'une femme douée d'une telle sensibilité pouvait se passer de quelque religion que ce soit. (p. 321)

En (10) et (11), la première personne désigne Alf le Crapaud dont le récit occupe les pages 167-172. En (17), la première personne désigne le narrateur sans nom auquel le nain Bergamme raconte son histoire. Ce qui l'atteste, c'est l'expression « toujours couché sur le dos ", qui décrit assez souvent Bergamme sur son lit d'hôpital. L'usage de je est donc ici l'usage déictique courant dans lequel la $1^{\text {ère }}$ personne désigne celui qui parle. Dans une conversation effective, celui qui parle est effectivement physiquement présent. Dans un roman, celui qui parle est le narrateur du récit en cours, récit global du texte du roman, ou récit partiel par un personnage promu au rang de narrateur dans le cours du roman, ce qui est le cas de Alf le Crapaud.

Dans l'incise 1 , les cas où la $1^{\text {ère }}$ personne est réalisée, soit comme sujet soit comme complément indirect du verbe de dire, sont les plus nombreux; il n'y a que quatre exemples dans lesquels il n'y a pas de $1^{\text {ère }}$ personne, (11), (13), (17), (19). Le relevé des exemples qui propose une $1^{\text {ère }}$ personne est donc superflu. On choisira quelques exemples pour pouvoir formuler un commentaire : 
1) «- Il débouche en ce moment douze cents personnes du faubourg de Rome, vient de me dire un de mes hommes, fit observer le lieutenant de gendarmerie, et ils poussent des cris de mort. "

2) De vivre sous le regard mort de tant de tableaux ne peut que rendre lucide, m'avait répondu Quevedo, avait continué Bergamme sans ouvrir les yeux ni bouger, et être lucide n'est-ce pas être cynique? (p. 100)

3) Ce qu'il n'a pas réussi, hélas! l'avais-je interrompu en jubilant, poursuit Bergamme. (p. 117)

4) Mais je ne connais pas de tableau moins élégant, moins «esthétique " que Guernica, m'avait interrompu Gerbraun de plus en plus amusé, continuait Bergamme. » (p. 118)

Dans ces quatre exemples, celui de Balzac et les trois premiers du roman de Rezvani, l'analyse est unique et conforte l'hypothèse 2 en approfondissant le paradoxe aperçu à travers l'analyse des temps verbaux. La $1^{\text {ère }}$ personne dans l'incise 1 doit être interprétée selon les règles de transfert propres à ce qu'on appelle couramment le discours direct introduit par une formule initiale ou par une incise de discours rapporté, cas qu'on trouve dans l'exemple (4):

4) Mais je ne connais pas de tableau moins élégant, moins "esthétique " que

Guernica, m'avait interrompu Gerbraun de plus en plus amusé, continuait Bergamme.» (p.

118)

Le je de " je ne connais pas de tableau moins élégant...», est interprété comme référant au sujet du verbe de communication « interrompre ", à savoir Gerbraun ; c'est là la règle de tranfert ordinaire de n'importe quel discours direct. On peut construire un exemple expérimental pour vérifier :

Je pars demain, annonça Marie. formes d'incises, celle à sujet inversé dont le modèle est " dit-il », celle à sujet non inversé et à la première personne, dont le modèle est « je crois » (1982: 81-147) : les premières, elle les appelle "incises de récit», les secondes "incises de discours». Les incises de discours sont disponibles dans tous les usages subjectifs du langage, aussi bien à l'oral qu'à l'écrit et elles ne sont pas spécifiques à la littérature romanesque.

Au contraire, les incises de récit sont propres au roman et ne se trouvent qu'à l'écrit. Elles sont, avec le style indirect libre, ce qui justifie le titre de l'ouvrage, "Unspeakable Sentences » traduit par "Phrases sans parole», c'est-à-dire phrases qu'on ne peut émettre dans les conditions des usages oraux du langage. Ces incises contiennent des verbes de parole (dire), de communication (raconter), de pensée (penser), de raisonnement (conclure). Elles ne connaissent ni la négation, ni l'interrogation. 

parole, lorsqu'il y a un verbe de dire, ou une conscience, lorsqu'il y a un verbe de penser. L'incise est un élément de l'appareil formel de l'énonciation, pour reprendre la formule de Benveniste. Elle est propre au roman parce que le roman a la propriété de permettre l'insertion dans l'usage objectif du langage, dénommée Histoire, d'usages subjectifs, c'està-dire de formes qui appartiennent au Discours : l'incise de récit sert à l'insertion de points de vues de personnages, par sa seule efficacité.

L'insertion de points de vue de personnages se fait canoniquement par l'introduction des paroles et des pensées des personnages, c'est pourquoi on considère à partir des cas de loin majoritaires que cette incise de récit introduit des discours rapportés ; c'est là une façon de rendre compte d'un point de vue, insérer des paroles attribuées à un personnage.

té que proposent l'exemple de Balzac et le roman de Rezvani consiste dans le fait que les points de vue introduits sont non seulement des paroles, ce qui est la forme de ce que dans notre représentation nous avons appelé « discours rapporté », mais aussi une nouvelle incise - celle appelée incise 1 dans notre représentation. Cette incise 1 réalise la mise en scène de l'introduction des paroles : l'incise 1 est introduite comme un nouveau point de vue par l'incise 2 .

$$
1 \text {, quelque chose - c'est le discours rapporté. }
$$

41 Finalement le personnage qui est montré en train de parler est celui qui est introduit dans l'incise 1, mais il l'est par un autre personnage, celui de l'incise 2.

On peut appeler le procédé ici inventé "roman à témoins", ou "roman par témoignages ». C'est essentiel dans le roman de Rezvani qui n'est formé que de comptesrendus de paroles tenues. C'est également essentiel dans La Rabouilleuse, puisque le moment où apparaît la forme relevée est celui de l'émeute à Issoudun, qui est décrite par les points de vue partiels des personnages qui apparaissent successivement pendant le déroulement de cette affaire particulièrement obscure.

\section{Les conséquences syntaxiques}

43 Du point de vue de la construction syntaxique, le choix de la $2^{\text {ème }}$ hypothèse pour rendre compte du phénomène dit de la double incise confirme les propriétés attribuables à la fonction syntaxique ici représentée.

Les incises sont, dans la structure de la phrase, des constituants qui ne sont ni souscatégorisés, ni sélectionnés par un quelconque constituant de la phrase: elles appartiennent aux ajouts, c'est-à-dire aux fonctions qui sont interprétées de façon constante quelle que soit la phrase dans laquelle elles sont insérées, à la différence des compléments dont l'interprétation dépend du terme qui les régit. Parmi ces ajouts, certains modifient l'interprétation référentielle de la phrase, par exemple les scéniques de temps ou de lieu; d'autres ne modifient pas l'interprétation référentielle, mais interviennent sur l'interprétation modale ou énonciative de la phrase : c'est le cas des incises.

45

Une propriété générale des ajouts est qu'ils sont itérables; d'une part, il peut y en avoir plusieurs, appartenant à des notions ou à des modalités distinctes - à la seule condition 
qu'il n'y ait pas de contradiction; d'autre part, certains ajouts peuvent être réalisés plusieurs fois moyennant des compatibilités entre eux :

Le lundi, s'il fait beau, je vais à la piscine.

Le lundi, le matin, je vais à la piscine.

La $1^{\text {ère }}$ hypothèse proposée pour l'analyse de la double incise aurait pour conséquence de construire les deux incises comme un constituant sur le modèle de la juxtaposition, donc de la simple itération d'un constituant identique, à interprétation identique : il aurait fallu calculer les compatibilités entre eux.

La $2^{\text {ème }}$ hypothèse en faisant appartenir l'incise1 à un constituant qui inclut le discours rapporté et l'incise 1, et l'incise 2 à un constituant plus inclusif, ou supérieur, dans la figure de l'arbre, qui inclut le premier constituant, distingue deux domaines syntaxiques qui correspondent aux deux domaines d'interprétation : le premier domaine peut être dit " point de vue 1 », le second « point de vue 2 ». Chacun contient une incise de récit.

Cette analyse conforte le principe de l'unicité des fonctions formulé dans Milner (1989: $376, n$.) :

«Une fonction donnée est représentée une seule fois dans un domaine ou réciproquement on doit toujours pouvoir définir un domaine tel que les fonctions y soient représentées une seule fois ».

et son application aux ajouts énonciatifs, qu'il appelle incidents, dans Marandin (1998: 46-47) :

«Il n'y a pas deux incidents de même type dans un domaine hôte donné. »

Les ajouts qui interviennent sur le statut énonciatif d'un énoncé ne sont pas itérables : le fait qu'il y en ait deux dans le cas observé de la double incise signifie qu'il y a deux domaines d'application, un pour l'incise 1 , un autre pour l'incise 2.

Ceci conduit à considérer que, dans la portée de l'incise 2, il y a autre chose que des paroles reproduites, ce qui éclaire le rôle des incises qui est d'introduire un nouvel énonciateur chaque fois qu'il en est besoin. Une des formes particulières que prend cette introduction est le discours rapporté au sens strict, une forme moins courante est celle représentée dans le corpus : l'énonciateur 2 introduit l'énonciateur 1, par une forme qui n'est pas un discours oral reproduit, mais une incise, c'est-à-dire une forme spécifique de l'écriture romanesque. Ce qu'on a appelé le discours rapporté n'est pas une reproduction, même fictive de discours oral, ce n'est pas un enregistrement, mais une création littéraire.

\section{BIBLIOGRAPHIE}

BANFIELD Ann (1982) Unspeakable Sentences. Narration and Representation in the Language of Fiction. (trad. Phrases sans parole. Théorie du récit et du style indirect libre, Paris : Le Seuil, 1995).

DELAVEAU Annie (1988) «La voix et les bruits : note sur les verbes introducteurs du discours rapporté », Linx 18. 
GOLDBERG Adèle (1995) A Construction Grammar Approach to Argument Structure, Chicago: The University of Chicago press.

HAMBURGER Kate (1986) Logique des genres littéraires. Paris : Le Seuil, (Die Logik der Dichtung, 1977).

MARANDIN Jean-Marie (1998) Grammaire de l'incidence, ms non publié.

MILNER Jean-Claude (1989) Introduction à une science du langage, Paris : Le Seuil.

\section{ANNEXES}

CORPUS

BALZAC, La Rabouilleuse, t.3, éd. Club du livre (p. 259) :

1) «-Il débouche en ce moment douze cents personnes du faubourg de Rome, vient de me dire un de mes hommes, fit observer le lieutenant de gendarmerie, et ils poussent des cris de mort. »

REZVANI, L'Origine du monde, Actes Sud (2000) :

2) De vivre sous le regard mort de tant de tableaux ne peut que rendre lucide, m'avait répondu Quevedo, avait continué Bergamme sans ouvrir les yeux ni bouger, et être lucide n'estce pas être cynique? (p. 100)

3) Ce qu'il n'a pas réussi, hélas ! l'avais-je interrompu en jubilant, poursuit Bergamme. (p. 117)

4) Mais je ne connais pas de tableau moins élégant, moins « esthétique » que Guernica, m'avait interrompu Gerbraun de plus en plus amusé, continuait Bergamme. » (p. 118)

5) « Exactement, m'étais-je écrié, continuait Bergamme, on ne critique pas Guernica!» (p.

119)

6) « Voyez-vous Gerbraun, avais-je continué, dit Bergamme, si Guernica n'avait pas ces dimensions, il y a longtemps que je l'aurais volé. » (p. 119)

7) « Bergamme est un petit homme tout à fait singulier, m'a dit Gerbraun, avait poursuivi Roberte, il veut tellement être le voleur des tableaux... » (p. 148)

8) ce Bergamme, c'était moi sans être moi, comprenez-vous Roberta ? m'avait dit Gerbraun, continuait Roberte. (p. 159)

9) Mais au contraire - et cela je ne l'ai compris que plus tard, a précisé mon jeune collaborateur Félix, continuait Alfle Crapaud - d'avoir été surpris avec Elise ... (p. 169)

10) « Mais non, jeune homme, lui avait dit Gerbraun, m'a encore rapporté mon collaborateur en hygrométrie, vous ne nous dérangez pas du tout!» (p. 170)

11) «Cesse de te plaindre, Gerbraun, avait dit Elise toujours allongée sous l'édredon, m'a encore rapporté mon jeune collaborateur. (p. 171)

12) Mais Quevedo, ce serait là un crime intellectuel impardonnable envers l'humanité ! avais-je crié en m'agitant, avait poursuivi Bergamme toujours couché immobile et les yeux fermés. (p. 188)

13) Une nuit, justement, avait poursuivi Quevedo, continuait Bergamme toujours étendu sur le dos, une nuit, le neveu de cette vieille bigote s'était mutilé atrocement. (p. 189)

14) Mais, dites-moi, Alf comment pouvez-vous faire subir de tels outrages à la Vie, lui avais-je demandé, le détestant violemment tout à coup, avait continué Bergamme. (p. 224)

15) Qui vous l'a dit Elise ? m'étais-je affolé, continuait Bergamme. (p. 282)

16) Et c'est comme ça, m'avait dit Roberte, avait continué avec presque joyeuse humeur Bergamme, oui c'est comme ça, m'avait-elle dit, que peu à peu les parois peintes commencèrent à se couvrir d'une légère moisissure... (p. 302) 
17) On aurait pu penser, ajoutait encore Quevedo, juste quelques instants avant que ne survienne le second « accident » du Grand Musée, m'avait dit Bergamme toujours couché sur le dos, oui, on aurait pu penser qu'une femme douée d'une telle sensibilité pouvait se passer de quelque religion que ce soit. (p. 321)

18) pour cela, nous devons principalement faire l'abandon de notre sexualité, soit volontairement en libérant nos femmes de leur embarrassant utérus, de sorte que la reproduction de notre espèce se fasse dans des sortes de cocotte - minute, disait-il par une sorte de plaisanterie, que je détestais, poursuivait Roberte, riant du vieux Crapaud ... (p. 343) 19) Ainsi nous, ces sortes de rats-taupes-glabres..., mais cependant humains, ces presque insectes... mais quand même humains serons-nous forcément obligés d'inventer un symbole représentant ce terrible manque, prétendait-il, avait dit Josette. (p. 343)

20) Oui, voilà ce que disait Félix ! m'avait affirmé Josette, poursuivait Bergamme toujours étendu sur le dos. (p. 361)

21) Bref, mon cher Bergamme, m'avait encore dit Gerbraun, avait poursuivi Bergamme gardant toujours les yeux fermés, bref, dès que je vous ai vu, mon très très cher Bergamme, vous m'avez mystérieusement plu parce que furieusement déplu. (p. 376)

22) Que voulez-vous, c'est ainsi, m'avait dit Gerbraun, avait poursuivi Bergamme, le Grand Musée a la charge de « conserver » à tout prix les chefs-d'œuvre... (p. 378)

23) Ne dites rien à personne, m'entendis-je crier à Gerbraun, continuait Bergamme, Dieu vous a mis dans son-mon dangereux secret ! (p. 396)

24) - C'est ce que vous auriez dû accomplir, lui avais-je répondu en grinçant d'un rire que j'entendais avec horreur sans pouvoir cependant le réprimer, continuait Bergamme, oui, avais-je dit à Gerbraun, c'est à ce moment -là qu'il était encore humainement possible de m'anéantir... (p. 397)

25) - Je n'ai nul besoin de vos justifications, Gerbraun, avais-je répondu calmement, continuait Bergamme toujours couché dans sa cellule, non ... (p. 401)

Les PERSONNES et les TEMPS

\begin{tabular}{|c|c|c|c|c|c|c|c|}
\hline & Incise 1 sujet & Incise 1 coi ou cod & Incise 2 sujet & Incise 2 coi & Incise 1 & Incise 2 & \\
\hline 1 & 3 & 1 & 3 & & présent & ps & 1 \\
\hline 2 & 3 & 1 & 3 & & pqpf & pqpf & 2 \\
\hline 3 & 1 & 3 & 3 & & pqpf & présent & 3 \\
\hline 4 & 3 & 1 & 3 & & pqpf & impft & 4 \\
\hline 5 & 1 & & 3 & & pqpf & impft & 5 \\
\hline 6 & 1 & & 3 & & pqpf & présent & 6 \\
\hline 7 & 3 & 1 & 3 & & $\mathrm{pc}$ & pqpf & 7 \\
\hline 8 & 3 & 1 & 3 & & pqpf & $\mathrm{impft}$ & 8 \\
\hline 9 & 3 & 1 & 3 & & $\mathrm{pc}$ & impft & 9 \\
\hline
\end{tabular}




\begin{tabular}{|c|c|c|c|c|c|c|c|}
\hline 10 & 3 & 3 & 3 & 1 & pqpf & pc & 10 \\
\hline 11 & 3 & & 3 & 1 & pqpf & pqpf & 11 \\
\hline 12 & 1 & & 3 & & pqpf & pqpf & 12 \\
\hline 13 & 3 & & 3 & & pqpf & impft & 13 \\
\hline 14 & 1 & 3 & 3 & & pqpf & pqpf & 14 \\
\hline 15 & 1 & & 3 & & pqpf & impf & 15 \\
\hline 16 & 3 & 1 & 3 & & pqpf & pqpf & 16 \\
\hline 17 & 3 & & 3 & 1 & $\mathrm{impft}$ & pqpf & 17 \\
\hline 18 & 3 & 1 & 3 & & $\mathrm{impft}$ & impft & 18 \\
\hline 19 & 3 & & 3 & & $\mathrm{impft}$ & pqpf & 19 \\
\hline 20 & 3 & 1 & 3 & & pqpf & impft & 20 \\
\hline 21 & 3 & 1 & 3 & & pqpf & pqpf & 21 \\
\hline 22 & 3 & 1 & 3 & & pqpf & pqpf & 22 \\
\hline 23 & 1 & 3 & 3 & & ps & impft & 23 \\
\hline 24 & 1 & 3 & 3 & & pqpf & impft & 24 \\
\hline 25 & 1 & & 3 & & pqpf & impft & 25 \\
\hline
\end{tabular}

\section{AUTEUR}

\section{ANNIE DELAVEAU}

Université de Paris 10 - Nanterre UMR 2329 MoDyCo 